\title{
Schizandrin A induces the apoptosis and suppresses the proliferation, invasion and migration of gastric cancer cells by activating endoplasmic reticulum stress
}

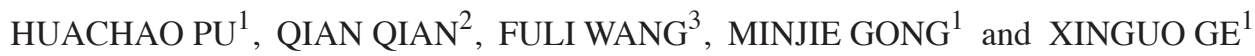 \\ ${ }^{1}$ Department of Oncology, Changzhou Hospital of Traditional Chinese Medicine; ${ }^{2}$ Department of Gastroenterology, \\ The First People's Hospital of Changzhou (The Third Affiliated Hospital of Soochow University); \\ ${ }^{3}$ Department of Oncology, Changzhou Jin Dongfang Hospital, Changzhou, Jiangsu 213000, P.R. China
}

Received March 23, 2021; Accepted August 2, 2021

DOI: $10.3892 / \mathrm{mmr} .2021 .12427$

\begin{abstract}
Apart from its basic antioxidant and anti-inflammatory properties, schizandrin A (SchA), which is isolated from Fructus schisandra, can exert anticancer effects on multiple cancer types. However, to the best of our knowledge, there has been no study identifying the impacts of SchA on gastric cancer (GC). Therefore, the aim of the present study was to identify how SchA functioned to affect the progression of GC. To investigate the role of SchA in GC development, Cell Counting Kit-8, colony formation, wound healing and Transwell assays were conducted to assess the viability, proliferation, migration and invasion of AGS cells, respectively. Then, the apoptosis rate and apoptosis- and endoplasmic reticulum (ER) stress-related protein expression levels in AGS cells exposed to SchA were detected via TUNEL assays and western blotting, respectively. Subsequently, the aforementioned functional assays were performed again in AGS cells exposed to both SchA and the ER stress inhibitor 4-phenylbutyric acid (4-PBA) for the confirmation of the effect of SchA on ER stress in GC. It was found that SchA markedly decreased the viability, proliferation, migration and invasion, while it induced the apoptosis of AGS cells. Moreover, the markers of ER stress were elevated by SchA treatment in AGS cells. Nevertheless, 4-PBA reversed the effects of SchA on the viability, proliferation, migration, invasion and apoptosis of AGS cells, accompanied by decreased expression of ER stress markers. In conclusion, the present study demonstrated that SchA induced the apoptosis and suppressed the proliferation, invasion and migration of GC cells by activating
\end{abstract}

Correspondence to: Dr Xinguo Ge, Department of Oncology, Changzhou Hospital of Traditional Chinese Medicine, 25 Heping Bei Lu, Tianning, Changzhou, Jiangsu 213000, P.R. China

E-mail: gexinguo20@163.com

Key words: schizandrin A, gastric cancer cells, endoplasmic reticulum stress, proliferation, invasion and migration
ER stress, which provides a theoretical basis for the use of SchA in the treatment of GC.

\section{Introduction}

Gastric cancer (GC) is one of the most common types of cancer with a high mortality and morbidity, and accounts for $\sim 1$ million deaths annually worldwide (1). Due to advances in diagnostic and therapeutic approaches, the incidence and mortality rate of GC in developed countries have decreased to relatively lower levels. In the United States, GC is the sixth leading cause of cancer-related deaths (2). However, GC is often diagnosed at an advanced stage, which increases the difficulty for doctors to effectively treat this disease (3). Therefore, it is important to identify novel treatment methods to improve the life quality of patients with GC.

Schizandrin A (SchA; Fig. 1A), isolated from Fructus schisandra, can exert a variety of therapeutic effects, including antioxidant, anti-inflammatory and anticancer effects (4). Accumulating evidence has demonstrated that SchA can suppress the proliferation, invasion and migration, and enhance the apoptosis of breast cancer cells by decreasing the expression levels of microRNA (miR)-155 (5). Additionally, it can promote the cell cycle arrest, apoptosis and cell death induced by heat shock transcription factor 1 , thereby impeding the proliferation of colorectal cancer cells (6). SchA can obstruct the proliferation and invasion of melanoma cells and the phosphorylation of PI3K/AKT by repressing $\mathrm{H} 19$ imprinted maternally expressed transcript expression (7). In addition, it can modulate the overactivation of Wnt and activate endoplasmic reticulum (ER) stress to induce the cell cycle arrest and apoptosis of triple-negative breast cancer cells (4). It has been shown that inhibition of IKK $\beta / N F-\kappa B$ signaling enhances the efficacy of gefitinib in the treatment of non-small cell lung cancer (4). Furthermore, a previous study reported the role of SchA in controlling the malignant behaviors of the TPC-1 thyroid cancer cell line by decreasing the expression levels of miR-429 (8). However, to the best of our knowledge, the effect of SchA on GC has not been investigated to date. 
Based on the hypothesis that SchA may affect the malignant behaviors of GC cells, SchA was purchased and used to treat GC cell suspensions. The present study investigated whether SchA could induce the apoptosis and suppress the proliferation, invasion and migration of GC cells by activating ER stress.

\section{Materials and methods}

Cell culture and drug preparation. SchA $\left(\mathrm{C}_{24} \mathrm{H}_{32} \mathrm{O}_{6}\right.$; cat. no. 61281-38-7) was procured from Shanghai Aladdin Biochemical Technology Co., Ltd. GES-1 human gastric epithelial cells and the AGS GC cell line, which were purchased from the American Type Culture Collection, were incubated in RPMI-1640 medium (Thermo Fisher Scientific, Inc.) supplemented with $10 \%$ FBS (Thermo Fisher Scientific, Inc.). The cells were cultured at $37^{\circ} \mathrm{C}$ in an incubator with $5 \% \mathrm{CO}_{2}$.

For the detection of ER stress, the ER stress inhibitor 4-phenylbutyric acid (4-PBA; Sigma-Aldrich; Merck KGaA) at a concentration of $7 \mathrm{mM}$ was used to pretreat AGS cells for $4 \mathrm{~h}$ at $37^{\circ} \mathrm{C}$, according to a previous study (9), to determine the role of SchA in ER stress.

Cell counting kit-8(CCK-8) assay. CCK-8 (MedChemExpress) was used to determine cell viability, according to the manufacturer's instructions. Briefly, AGS cells were seeded at a density of $4 \times 10^{3}$ cells/well in 96-well plates and cultured. SchA at concentrations of $10,20,30,40$ and $50 \mu \mathrm{M}$ was administered to the cells, as previously described $(4-7,10)$. After incubation at $37^{\circ} \mathrm{C}$ for the corresponding time intervals $(24,48$ and $72 \mathrm{~h}$ ), $10 \mu \mathrm{lCCK}-8$ was added to the cells at $37^{\circ} \mathrm{C}$ for $4 \mathrm{~h}$ to detect cell viability. The optical density at $450 \mathrm{~nm}$ was measured using a microplate reader (Thermo Fisher Scientific, Inc.).

Colony formation assay. AGS cells were seeded onto 6-well plates (500 cells per well) with or without treatment of SchA at different concentrations $(10,20$ and $30 \mu \mathrm{M})$ for $24 \mathrm{~h}$ and/or 4-PBA ( $\left.7 \mathrm{mM}, 37^{\circ} \mathrm{C}, 4 \mathrm{~h}\right)$, and cultured at $37^{\circ} \mathrm{C}$ for 2 weeks. Following fixation with $3.7 \%$ paraformaldehyde at room temperature for $20 \mathrm{~min}$, the cell colonies were stained with $0.05 \%$ crystal violet solution at room temperature for $30 \mathrm{~min}$. The macroscopic cell group formed by the continuous proliferation of a single cell in vitro for more than six generations was regarded as a clone, and cells were counted manually under an inverted optical microscope (magnification, x10; Olympus Corporation).

Wound healing assay. For the evaluation of cell migration, cells were incubated in 96-well plates at a density of $4 \times 10^{5}$ cells per well. A scratch was created in the cell monolayer using a $200-\mu 1$ pipette tip. After culturing in complete medium at $37^{\circ} \mathrm{C}$ for 0 and $24 \mathrm{~h}$, the plates and the wound closure were visualized using a light microscope (magnification, x100; Nikon Corporation). The cell migration rate was analyzed using Image-Pro Plus 6.0 software (Media Cybernetics, Inc.).

Transwell assay. All cells were inoculated at a density of $2 \times 10^{4}$ cells/well in the upper chamber of Transwell plates
(8- $\mu \mathrm{m}$ pore size; Corning, Inc.). The surface of the upper chamber was precoated with Matrigel ${ }^{\mathrm{TM}}$ (BD Biosciences) at $37^{\circ} \mathrm{C}$ for $1 \mathrm{~h}$. The FBS-free RPMI-1640 medium was added in the upper chamber, and $500 \mu \mathrm{l}$ fresh medium containing $10 \%$ FBS was added in the lower chamber. Cells were incubated at $37^{\circ} \mathrm{C}$ with $5 \% \mathrm{CO}_{2}$ for $24 \mathrm{~h}$, followed by staining using $0.1 \%$ crystal violet solution at room temperature for $20 \mathrm{~min}$. Images were obtained using a light microscope (magnification, x100; Nikon Corporation).

Western blotting. Total protein was extracted from cells under different culture conditions using RIPA lysis buffer (Sigma-Aldrich; Merck KGaA) according to the manufacturer's protocol. A BCA assay kit (Beyotime Institute of Biotechnology) was used for the quantification of total protein. Proteins (30 $\mu \mathrm{g}$ per lane) were separated via $12 \%$ SDS-PAGE and then transferred to PVDF membranes. After blocking in 5\% skimmed milk at room temperature for $2 \mathrm{~h}$, membranes were incubated with antibodies against MMP-2 (cat. no. ab92536; 1:1,000; Abcam), MMP-9 (cat. no. ab76003; 1:1,000; Abcam), E-cadherin (cat. no. ab40772; 1:10,000; Abcam), $\mathrm{N}$-cadherin (cat. no. ab76011; 1:5,000; Abcam), vimentin (cat. no. ab92547; 1:1,000; Abcam), Bcl-2 (cat. no. ab32124; 1:1,000; Abcam), Bax (cat. no. ab32503; 1:1,000; Abcam), cleaved-caspase 3 (cat. no. ab32042; 1:500; Abcam), cleaved-poly (ADP-ribose) polymerase (PARP; cat. no. ab32064; 1:1,000; Abcam), caspase 3 (cat. no. ab184787; 1:2,000; Abcam), PARP (cat. no. ab191217; 1:1,000; Abcam), heat shock protein family A (Hsp70) member 5 (HSPA5; cat. no. ab108615; 1:1,000; Abcam), phosphorylated (p)-PERK (cat. no. MA5-15033; 1:1,000; Thermo Fisher Scientific, Inc.), p-eukaryotic initiation factor $2 \alpha$ (p-eIF $2 \alpha$; cat. no. 3398; 1:1,000; Cell Signaling Technology, Inc.), CHOP (cat. no. PA5-28956; 1:1,000; Thermo Fisher Scientific, Inc.), PERK (cat. no. ab79483; 1:1,000; Abcam), eIF2 $\alpha$ (cat. no. 5324; 1:1,000; Cell Signaling Technology, Inc.) and GAPDH (cat. no. ab9485; 1:2,500; Abcam) overnight at $4^{\circ} \mathrm{C}$, and then a Goat Anti-Rabbit IgG H\&L (HRP) secondary antibody (cat. no. ab6721; 1:2,000; Abcam) was added for $1 \mathrm{~h}$ at room temperature. An electrochemical luminescence reagent (MilliporeSigma) was applied for the visualization of the bands. The grey region of bands was semi-quantified using ImageJ software (v1.8.0; National Institutes of Health).

TUNEL assay. Apoptosis was analyzed using a TUNEL Apoptosis Detection kit (Beyotime Institute of Biotechnology) according to the manufacturer's protocol. The cells were fixed in $4 \%$ paraformaldehyde at room temperature for $0.5 \mathrm{~h}$ and then washed in PBS twice. After incubation with TUNEL reaction mixture for $1 \mathrm{~h}$ at $37^{\circ} \mathrm{C}$ in the dark, cells were washed with PBS and incubated with a converter-peroxidase reagent for $30 \mathrm{~min}$. The nuclei were counterstained with DAPI for $5 \mathrm{~min}$ at room temperature in the dark. Subsequently, diaminobenzidine solution was used to treat the cells, and five fields of view were randomly selected to capture cell images using a fluorescence microscope (magnification, x100; Nikon Corporation) and analyzed using Image Pro Plus 6.0 software (Media Cybernetics, Inc.). 
A<smiles>COc1cc2c(c(OC)c1OC)-c1c(cc(OC)c(OC)c1OC)C[C@@H](C)[C@@H](C)C2</smiles>

D

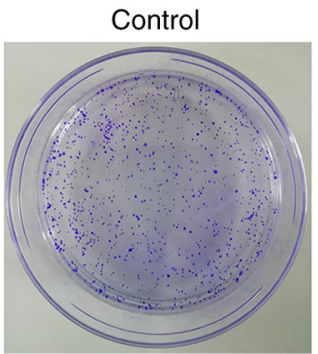

B

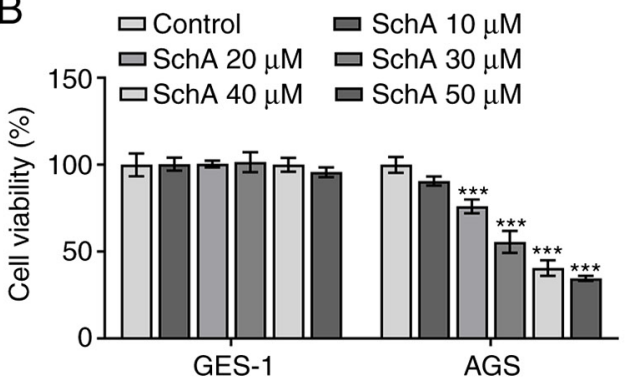

$\operatorname{SchA} 10 \mu \mathrm{M}$

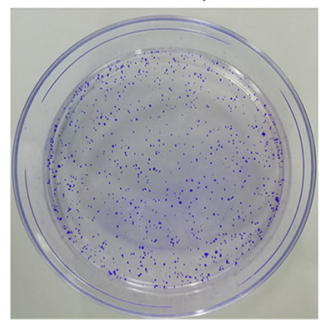

$\operatorname{SchA} 20 \mu \mathrm{M}$

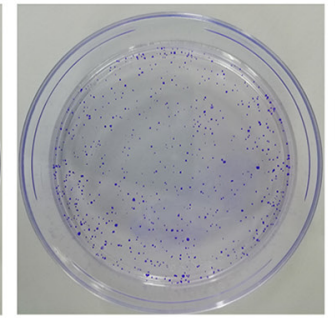

C

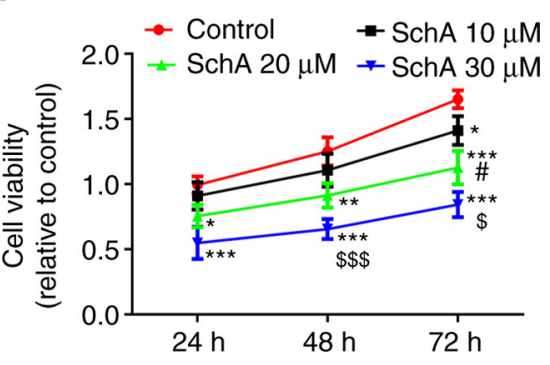

$\operatorname{SchA} 30 \mu \mathrm{M}$

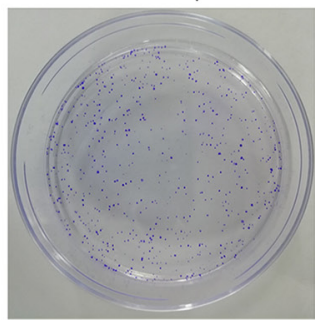

Figure 1. SchA decreases the viability of GC cells. (A) The chemical structure of SchA. (B) The viability of AGS cells affected by SchA at concentrations of $10,20,30,40$ and $50 \mu \mathrm{M}$. (C) The viability of AGS cells affected by SchA at concentrations of 10,20 and $30 \mu \mathrm{M}$. ${ }^{*} \mathrm{P}<0.05,{ }^{* * *} \mathrm{P}<0.01$ and ${ }^{* * * *} \mathrm{P}<0.001$ vs. Control; ${ }^{\#} \mathrm{P}<0.05$ vs. $10 \mu \mathrm{M} \mathrm{SchA} ;{ }^{\$} \mathrm{P}<0.05$ and ${ }^{\$ \$}{ }^{\$} \mathrm{P}<0.001$ vs. $20 \mu \mathrm{M}$ SchA. (D) The colony formation of AGS cells after SchA exposure. SchA, schizandrin A.

Statistical analysis. Statistical analysis was performed using GraphPad Prism 6.0 (GraphPad Software, Inc.) and SPSS 22.0 (IBM Corp.) software. Continuous variables are presented as the mean \pm SD. P-values were calculated using unpaired Student's t-test for comparisons between two groups or one-way ANOVA with Tukey's test for comparisons among multiple groups. $\mathrm{P}<0.05$ was considered to indicate a statistically significant difference.

\section{Results}

SchA suppresses the viability, proliferation, invasion and migration of GC cells. To investigate the role of SchA in the functions of GC cells, GES-1 and AGS cells were treated with different concentrations of SchA. As shown in Fig. 1B, the viability of GES-1 cells was not affected by SchA, while increasing concentrations of SchA gradually reduced the viability of AGS cells. It was further demonstrated that the viability of AGS cells was decreased in a dose- and time-dependent manner (Fig. 1C). Next, the effects of SchA on the proliferation, invasion and migration of GC cells were measured, and the results indicated that SchA exerted inhibitory effects on the proliferation (Fig. 1D), migration (Fig. 2A) and invasion (Fig. 2B) of AGS cells in a dose-dependent manner. The expression levels of MMP-2 and MMP-9 were also decreased by SchA treatment in AGS cells (Fig. 2C). The expression level of E-cadherin was increased, while the expression levels of $\mathrm{N}$-cadherin and vimentin were decreased by SchA treatment in AGS cells (Fig. 2D).

SchA induces the apoptosis of AGS cells. The apoptosis of AGS cells exposed to SchA was subsequently detected to determine the role of SchA in this process. Notably, the apoptosis of AGS cells was enhanced by increasing doses of SchA when compared with the control group (Fig. 3A). Similarly, the expression levels of the anti-apoptotic protein Bcl-2 were downregulated, while those of the pro-apoptotic proteins Bax, cleaved-caspase 3/caspase 3 and cleaved-PARP/PARP were increased after SchA treatment in AGS cells (Fig. 3B). However, the expression levels of caspase 3 and PARP remained unchanged after exposure to $10 \mu \mathrm{M}$ SchA in AGS cells, while they were markedly decreased as the doses of SchA increased to 20 and $30 \mu \mathrm{M}$. Overall, it was suggested that SchA induced the apoptosis of AGS cells.

SchA activates ER stress in AGS cells. Stimulating factors, which can disrupt the homeostasis of proteins, can induce ER stress in tumor cells (11). The expression level of HSPA5, the marker of ER stress, was increased by SchA in AGS cells (Fig. 4A). Increasing doses of SchA increased the phosphorylation of eIF2 $\alpha$ and PERK, as well as the expression levels of CHOP, indicating the activation of ER stress by SchA in AGS cells (Fig. 4B). Since $30 \mu \mathrm{M}$ SchA could notably activate ER stress in AGS cells, this dose was selected for the subsequent experiments.

4-PBA, an ER stress inhibitor, reverses the anti-proliferative, anti-invasive, anti-migratory and pro-apoptotic effects of SchA on AGS cells. To further determine whether SchA affects the functions of AGS cells, the ER stress inhibitor 4-PBA was used to treat AGS cells. Cell viability was decreased after treatment with SchA, which was restored by 4-PBA (Fig. 5A). The proliferation, migration and invasion of AGS cells were suppressed by SchA, while co-treatment with 4-PBA and SchA alleviated this effect (Fig. 5B-D). The expression levels of MMP-2 and MMP-9, which are associated with invasion and cancer angiogenesis (12), were 
A

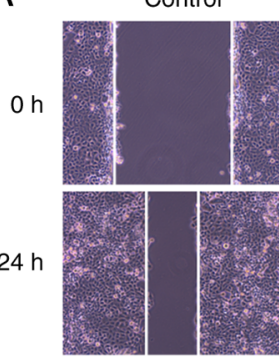

B

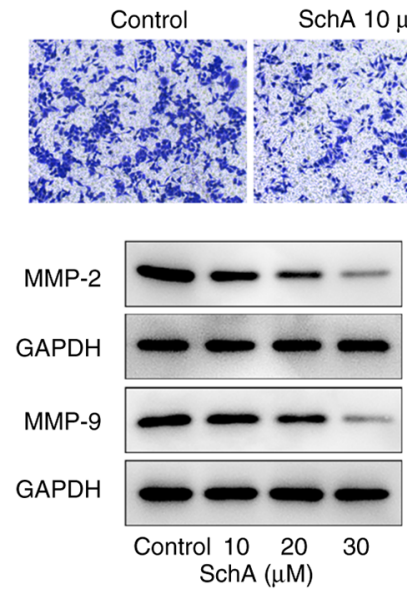

$\operatorname{SchA} 10 \mu \mathrm{M}$
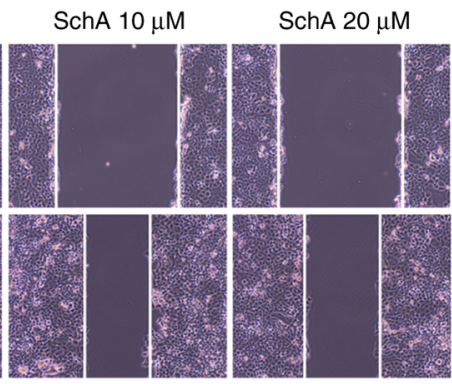

$\operatorname{SchA} 20 \mu \mathrm{M}$
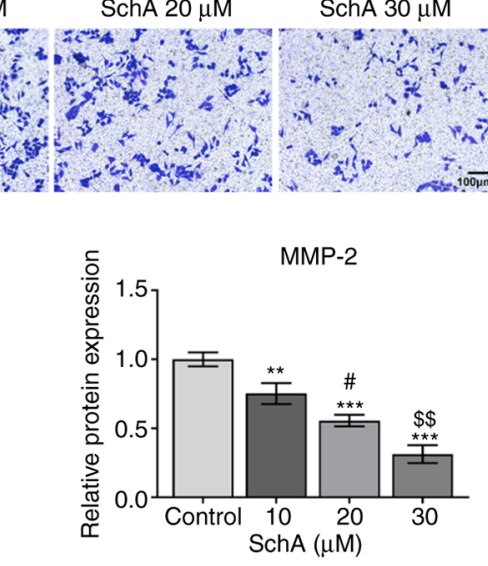

SchA $30 \mu \mathrm{M}$

SchA $30 \mu \mathrm{M}$
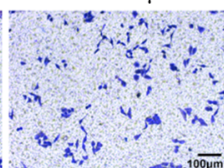
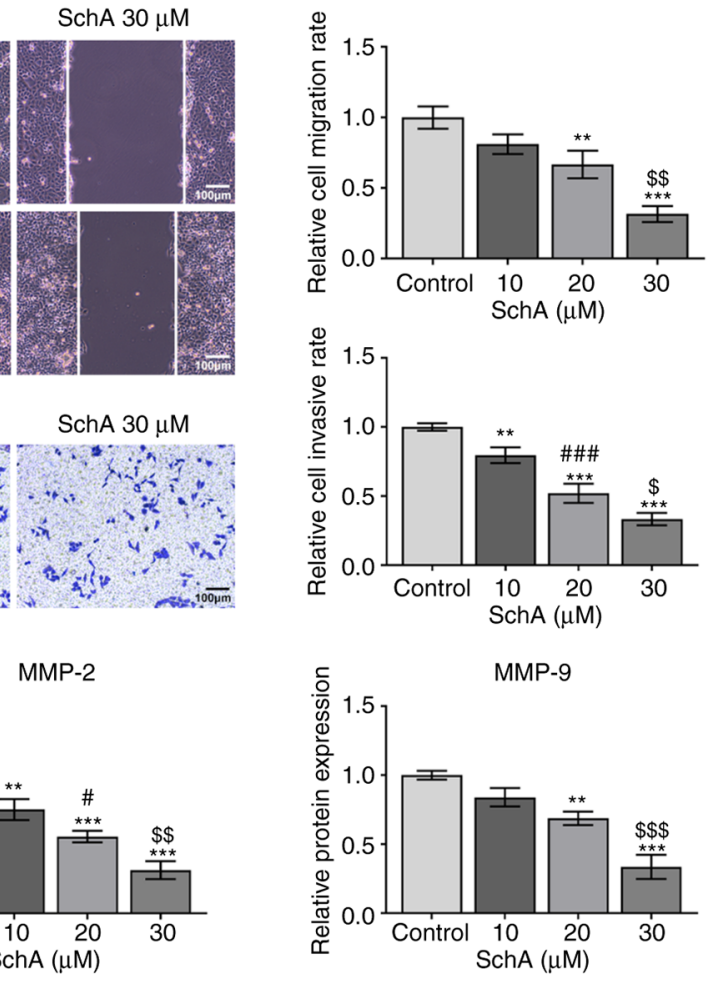

D
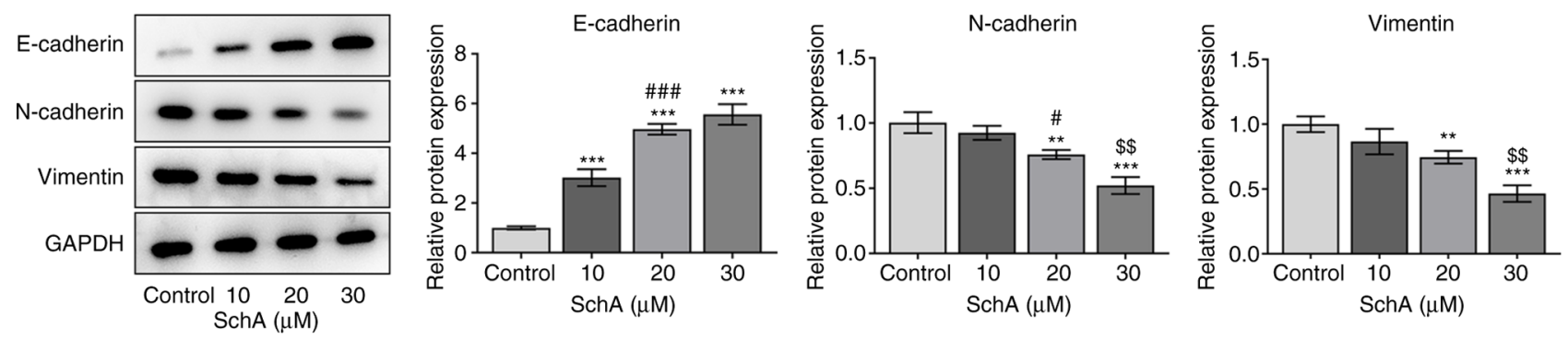

Figure 2. SchA suppresses the proliferation, invasion and migration of GC cells. The (A) migration and (B) invasion of AGS cells after SchA exposure. (C) The expression levels of MMP-2 and MMP-9 were determined via western blotting. (D) The expression levels of epithelial-mesenchymal transition-related proteins were determined via western blotting. ${ }^{* * *} \mathrm{P}<0.01$ and ${ }^{* * * *} \mathrm{P}<0.001$ vs. Control; ${ }^{\#} \mathrm{P}<0.05$ and ${ }^{\# \# \#} \mathrm{P}<0.001$ vs. $10 \mu \mathrm{M} \mathrm{SchA} ;{ }^{\$} \mathrm{P}<0.05,{ }^{\$ \$} \mathrm{P}<0.01$ and ${ }^{\$ \$ \$} \mathrm{P}<0.001$ vs. $20 \mu \mathrm{M}$ SchA. SchA, schizandrin A.

found to be increased by co-treatment with 4-PBA and Sch A compared with those in the SchA group (Fig. 5E). The expression of E-cadherin was downregulated, while expression levels of $\mathrm{N}$-cadherin and vimentin were upregulated in the 4-PBA + SchA group compared with that in the SchA group (Fig. 5F). The apoptosis of AGS cells was induced by SchA, while co-treatment of cells with 4-PBA and SchA led to decreased levels of apoptosis compared with the SchA group (Fig. 6A and B). In addition, the results of western blotting demonstrated that HSPA5 expression was increased by SchA treatment, but was reversed by 4-PBA treatment (Fig. 6C). Moreover, the expression levels of p-PERK, p-eIF $2 \alpha$ and CHOP were increased by SchA treatment, while these levels were decreased by 4-PBA and SchA treatment, suggesting that 4-PBA inhibited ER stress, which was activated by SchA (Fig. 6D). These results suggested the important role of 4-PBA in reversing the anti-proliferative, anti-invasive, anti-migratory and pro-apoptotic effects of SchA on AGS cells.

\section{Discussion}

GC is one of the most prevalent types of cancer and, at present, it remains difficult to completely cure (13). Surgical interventions, which are essential methods for cancer treatment, have increased the overall survival rates of patients with GC (14). However, the prognosis of $\mathrm{GC}$ remains poor, and the majority of patients with GC are diagnosed at an advanced stage (15). In recent years, the active ingredients of traditional Chinese medicine (TCM) have been demonstrated to serve critical roles in the treatment of cancer, since the combined use of chemotherapy and TCM can greatly improve the overall survival of patients with GC (16).

Sch is the fruit of Schisandra chinensis and is a type of TCM used as a food supplement, as well as for medical interventions (17). In addition to its use in the treatment of clinical symptoms, such as fatigue, cough, dysentery and insomnia, it has been investigated for its notable antioxidant and antiviral effects in recent years (18). Accumulating evidence 
A

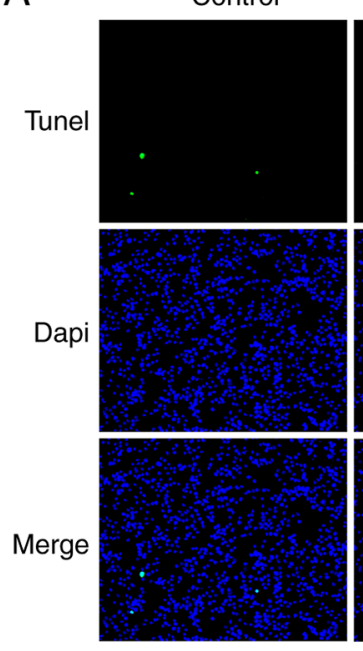

B

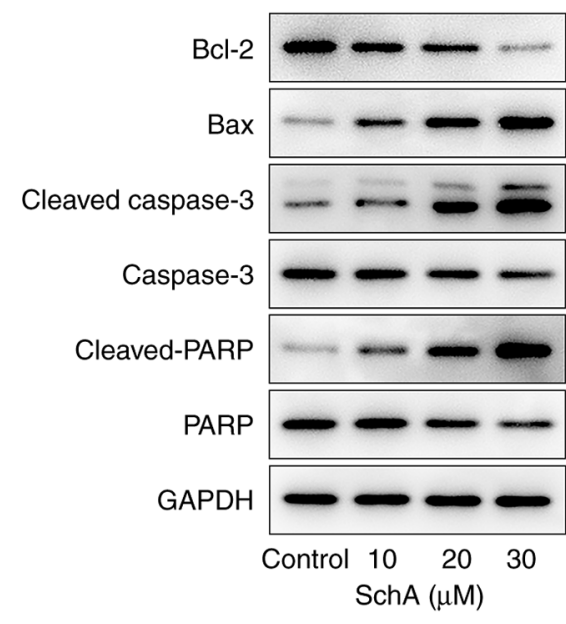

SchA $10 \mu \mathrm{M}$
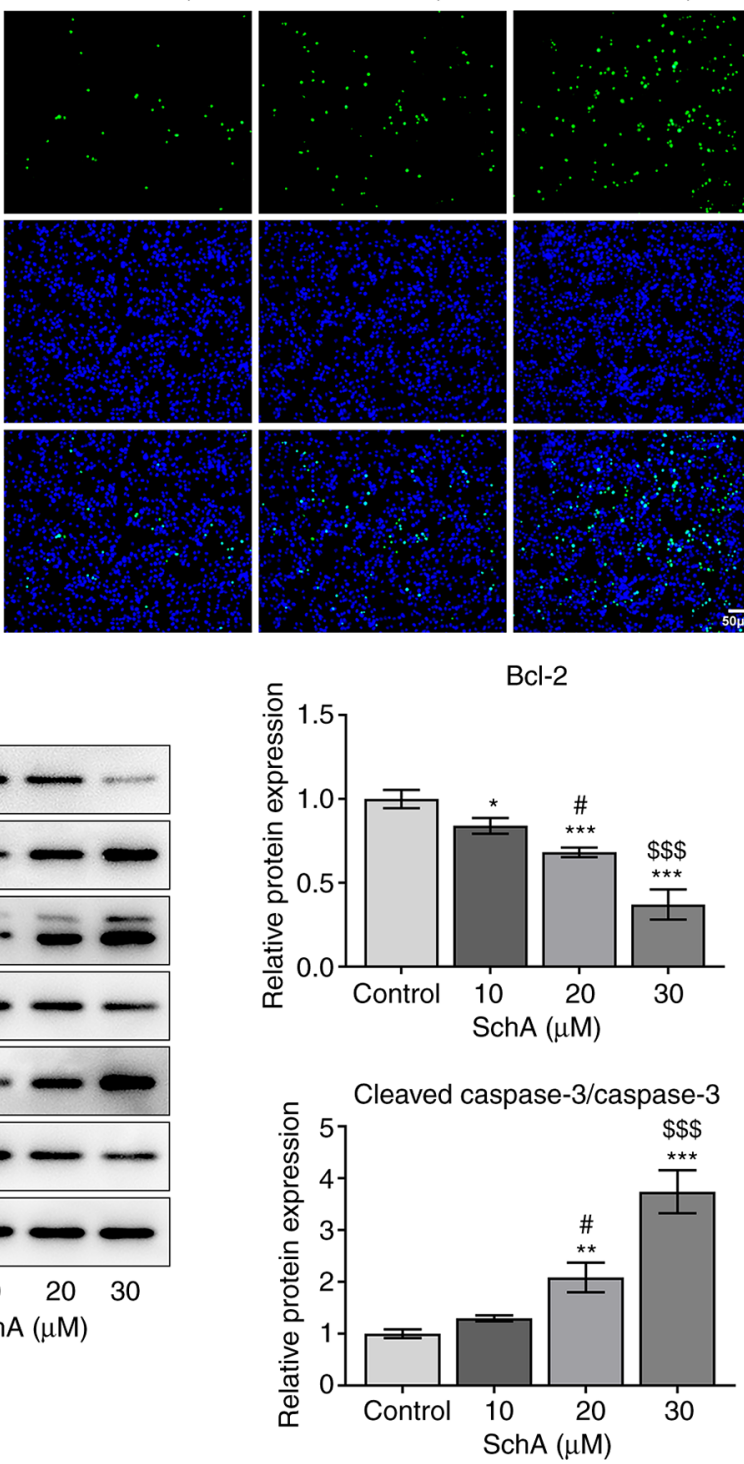
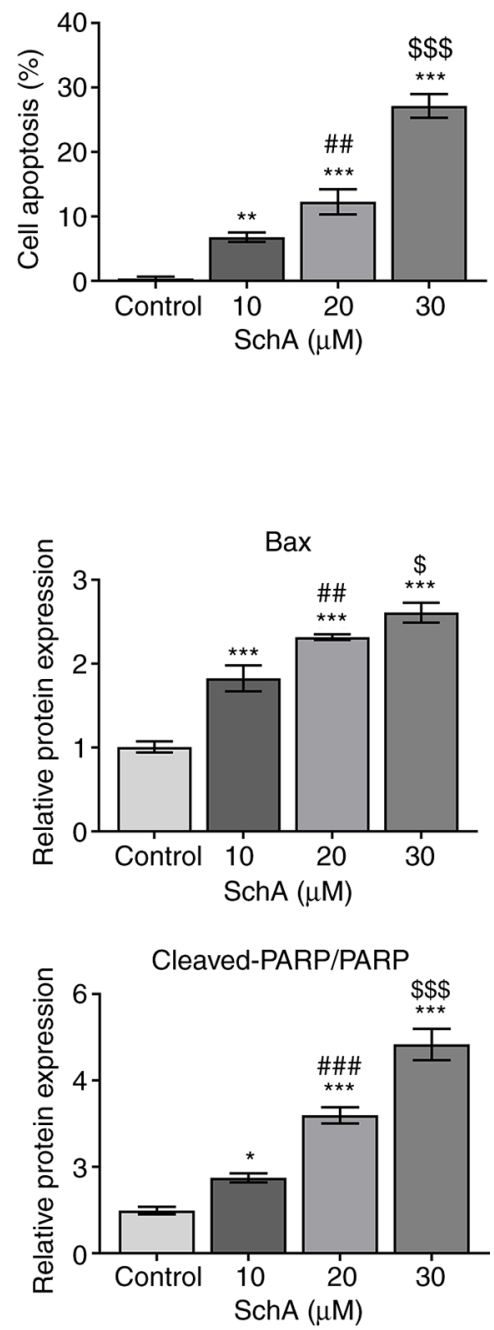

Figure 3. SchA induces the apoptosis of AGS cells. (A) The apoptosis of AGS cells after SchA exposure. (B) The expression levels of apoptosis-related proteins in AGS cells after SchA exposure. ${ }^{*} \mathrm{P}<0.05,{ }^{* * *} \mathrm{P}<0.01$ and ${ }^{* * *} \mathrm{P}<0.001$ vs. Control; ${ }^{\#} \mathrm{P}<0.05,{ }^{\# \#} \mathrm{P}<0.01$ and ${ }^{\# \# \#} \mathrm{P}<0.001$ vs. $10 \mu \mathrm{M} \mathrm{Sch}$; ${ }^{\$} \mathrm{P}<0.05$ and ${ }_{\$ \$} \mathrm{P}<0.001$ vs. $20 \mu \mathrm{M}$ SchA. SchA, schizandrin A; PARP, poly(ADP-ribose) polymerase.

has indicated that the cytotoxicity of SchA can suppress the proliferation of human cancer cells, thereby inducing their apoptosis and delaying cancer progression (19). The present study first revealed that the viability of AGS cells was affected by increasing doses of SchA, and the results of the colony formation assay demonstrated the reduced colony-forming ability of AGS cells upon SchA exposure. Moreover, the migration and invasion of AGS cells were both decreased after SchA treatment, and SchA at the concentration of $30 \mu \mathrm{M}$ exerted the most obvious inhibitory effects on the migration and invasion of AGS cells. Furthermore, the apoptosis of AGS cells was significantly induced by SchA, which was in line with previous findings suggesting the promoting role of SchA in the apoptosis of human cancer cells (7).

The ER is the organelle where protein handling, modification and folding occur (20). The homeostasis of the ER is essential for cell functions and cell fate (20). However, upon stimulation by certain environmental factors, the homeostasis of the ER cannot be maintained, thereby triggering ER stress (21). ER stress has been well-documented to be involved in the pathophysiology of most common diseases, including metabolic disease, neurodegenerative disease, inflammatory disease and cancer (22). ER stress, which is associated with the form of heat shock protein 70-type binding immunoglobulin protein/HSPA5, activates intraluminal ER sensors, including eIF2 $\alpha$, PERK and activating transcription factor 6 (23). p-eIF2 $\alpha$ suppresses the commencement of protein synthesis, which reduces the protein loading in the ER and ameliorates ER stress (24). PERK serves as a critical signaling protein related to the evolution of ER stress, and it is considered to block the aggregation of unfolded proteins in the ER, thereby modulating ER stress in an adverse feedback pattern (25). $\mathrm{CHOP}$ is considered as a key protein responsible for the modulation of ER stress-mediated cell apoptosis (26). A previous study reported that ER stress hyperactivated both 
A

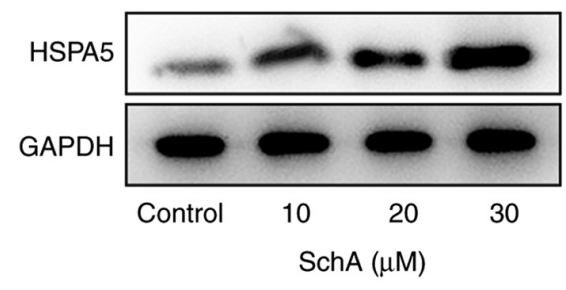

B

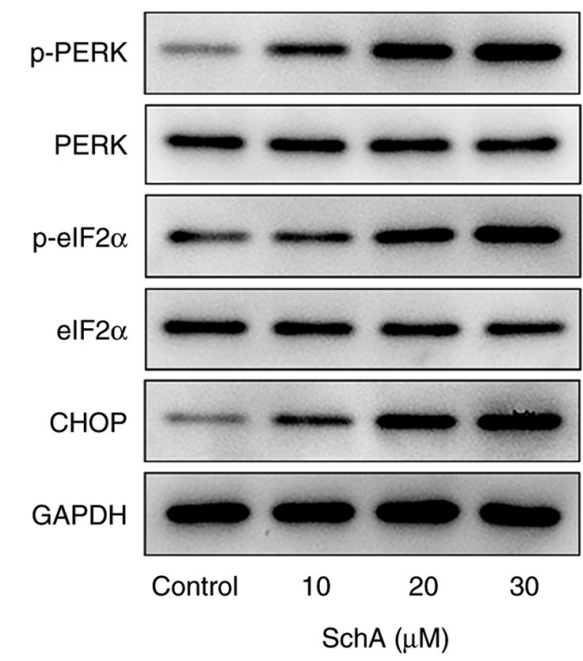

p-elF2 $\alpha /$ elF2 $\alpha$

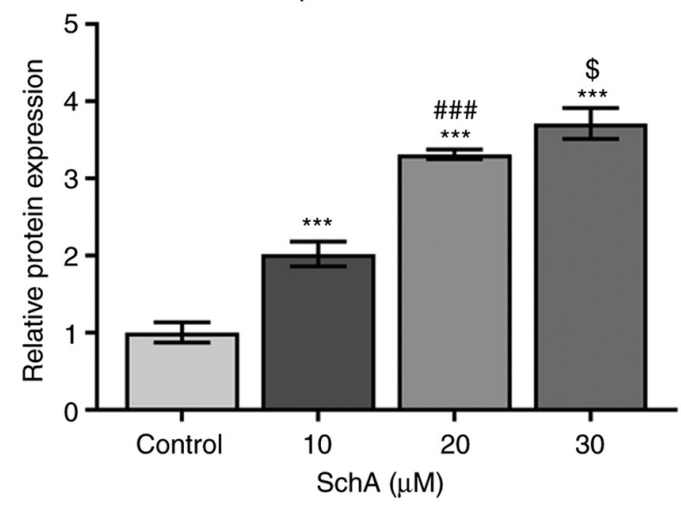

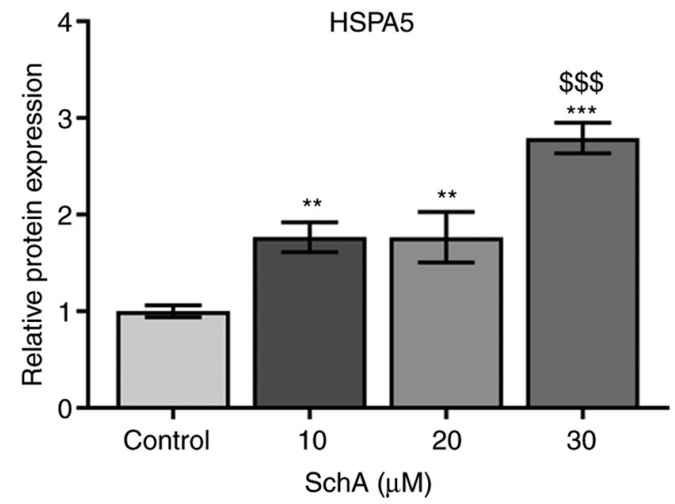

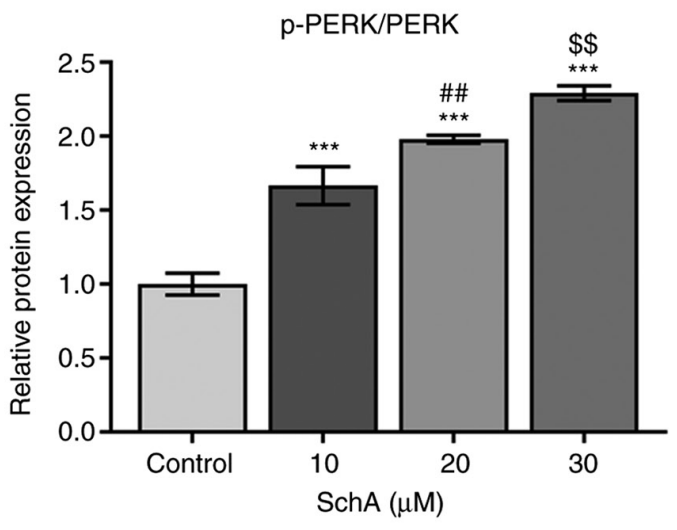

CHOP

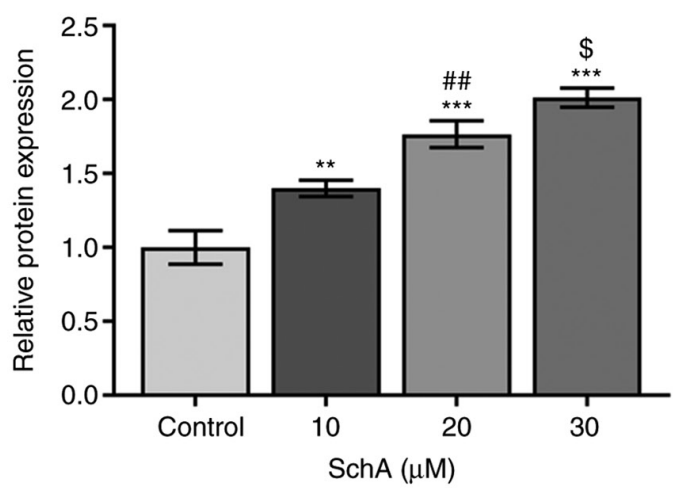

Figure 4. SchA activates ER stress in AGS cells. (A) The expression level of the marker of ER stress (HSPA5). (B) The expression levels of markers related to ER stress. ${ }^{* *} \mathrm{P}<0.01$ and ${ }^{* * * *} \mathrm{P}<0.001$ vs. Control; ${ }^{\# \#} \mathrm{P}<0.01$ and ${ }^{\# \# \#} \mathrm{P}<0.001$ vs. $10 \mu \mathrm{M} \mathrm{SchA} ;{ }^{\$} \mathrm{P}<0.05,{ }^{\$ \$} \mathrm{P}<0.01$ and ${ }^{\$ \$ \$} \mathrm{P}<0.01$ vs. $20 \mu \mathrm{M}$ SchA. ER, endoplasmic reticulum; HSPA5, heat shock protein family A (Hsp70) member 5; p-, phosphorylated; eiF2 $\alpha$, eukaryotic initiation factor $2 \alpha$; SchA, schizandrin A.

PERK and inositol-requiring transmembrane kinase/endoribonuclease $1 \alpha(\operatorname{IRE}-1 \alpha)$, contributing to the entry into the apoptosis pathway (27). In the present study, SchA triggered the apoptosis of AGS cells and phosphorylation of PERK and IRE-1 $\alpha$. Therefore, it was hypothesized that SchA may affect the behaviors of AGS cells via the activation of ER stress. These results suggested that the phosphorylation of PERK and eIF2 $\alpha$, and elevated expression levels induced by SchA were reversed by 4-PBA, which verified this hypothesis.

Studies have shown that the ER stress response was ubiquitous in tumor tissues, and that it regulates the occurrence and development of tumors, and participates in tumor invasion and metastasis (28-31). The degree of ER stress is positively correlated with the depth of invasion and the degree of metastasis (32). Li et al (33) revealed that downregulation of heparinase can reverse ER stress-mediated invasion of breast cancer cells. Moreover, Liu et al (34) observed that knocking down the expression of HSPA5, an indicator protein of ER stress, could significantly reduce the invasive ability of tumor cells by inhibiting the PI3K/AKT signaling pathway. These studies suggest that ER stress may be an important inducement for tumor cell invasion, and could play a key role in tumor metastasis. The invasion and migration of gastric cancer cells are significantly enhanced after treatment with tunicamycin (an ER stress inducer), indicating that 


\section{A}

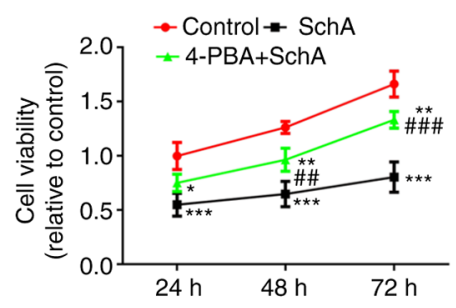

B
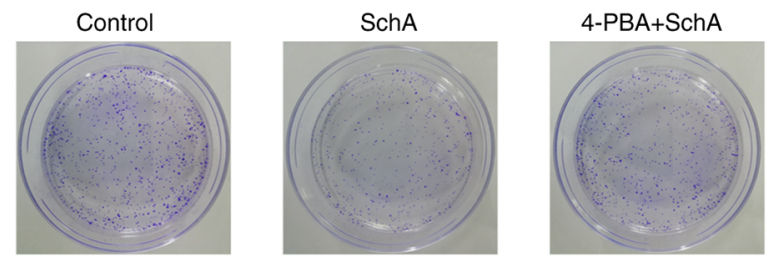

C
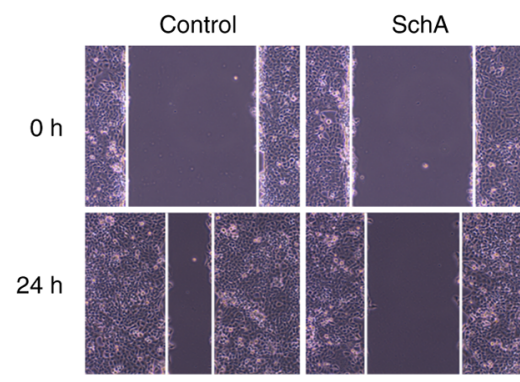

4-PBA+SchA

D

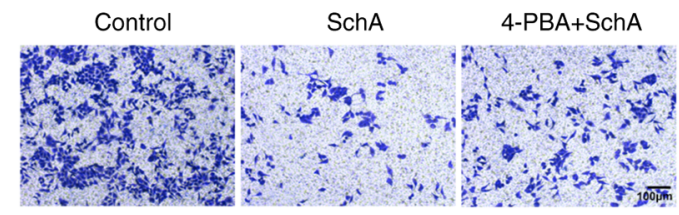

E
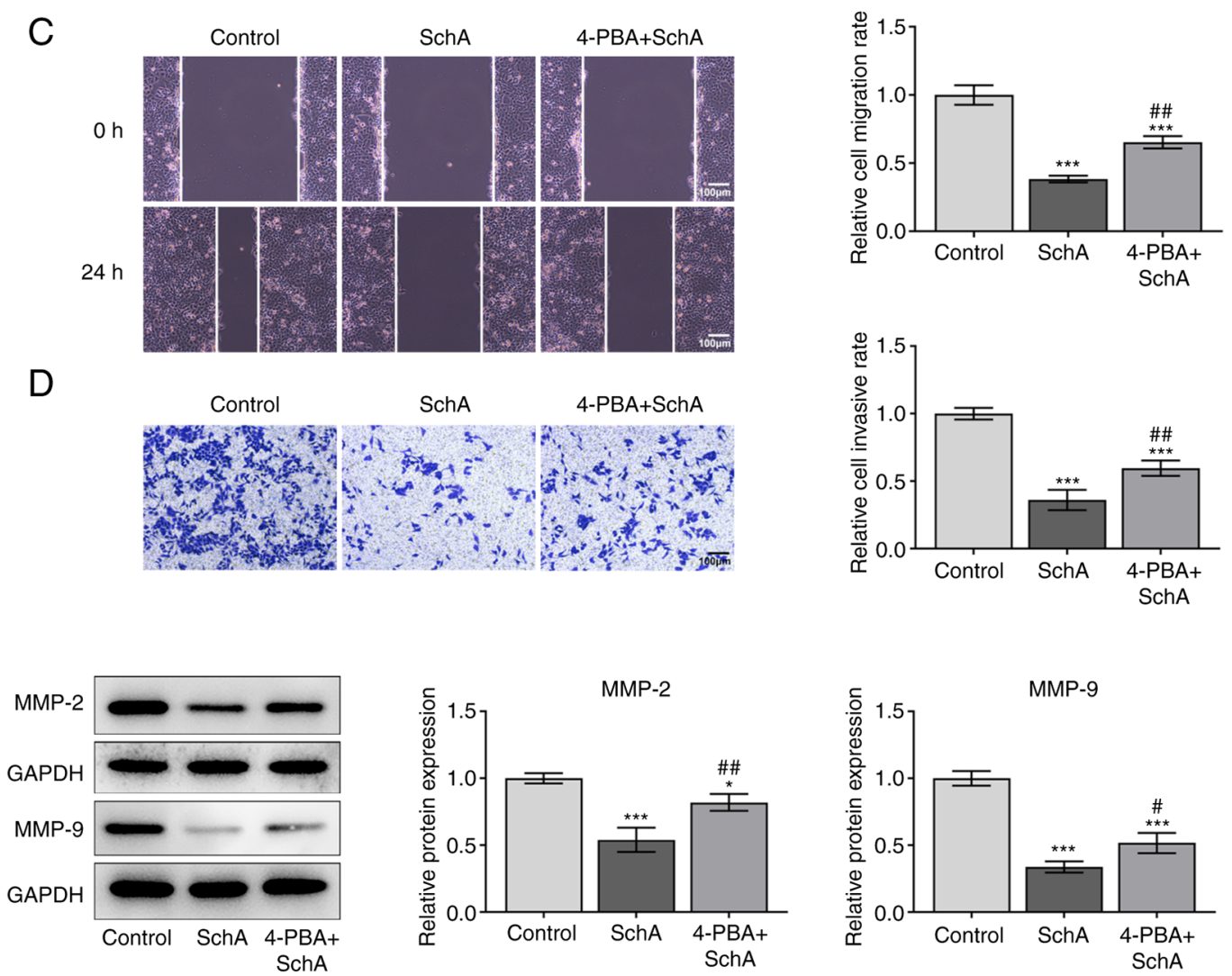
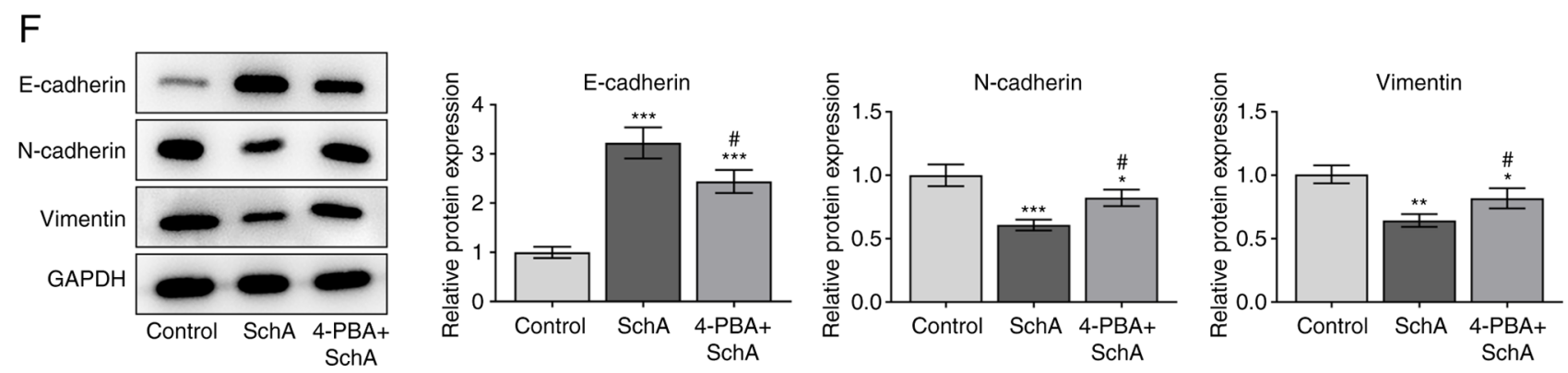

Figure 5.4-PBA, an endoplasmic reticulum stress inhibitor, reverses the anti-proliferative, anti-invasive and anti-migratory effects of SchA in AGS cells. (A) The viability of AGS cells was affected by SchA and 4-PBA treatment. The (B) colony formation, (C) migration and (D) invasion of AGS cells after SchA and 4-PBA exposure. (E) The expression levels of MMP-2 and MMP-9 were determined via western blotting. (F) The expression levels of epithelial-mesenchymal transition-related proteins were determined via western blotting. ${ }^{*} \mathrm{P}<0.05,{ }^{* * *} \mathrm{P}<0.01$ and ${ }^{* * * *} \mathrm{P}<0.001$ vs. Control; ${ }^{\#} \mathrm{P}<0.05,{ }^{\# \#} \mathrm{P}<0.01$ and ${ }^{\# \# \#} \mathrm{P}<0.001$ vs. SchA. SchA, schizandrin A; 4-PBA, 4-phenylbutyric acid.

ER stress promotes the invasion and migration of gastric cancer cells (35). Thus, ER stress may be an important regulatory mechanism of gastric cancer metastasis. In the present study, 4-PBA reversed the anti-proliferative, anti-invasive, anti-migratory and pro-apoptotic effects of SchA on AGS cells. However, a limitation of the present study is the lack of flow cytometry assays to further confirm the apoptosis rates. Furthermore, the downstream regulators of SchA in the regulation of ER stress are still unknown, and thus will be investigated in the future.
In conclusion, the present study demonstrated that SchA induced the apoptosis and suppressed the proliferation, invasion and migration of GC cells by activating ER stress, providing a theoretical basis for the use of SchA in the treatment of GC.

\section{Acknowledgements}

Not applicable. 

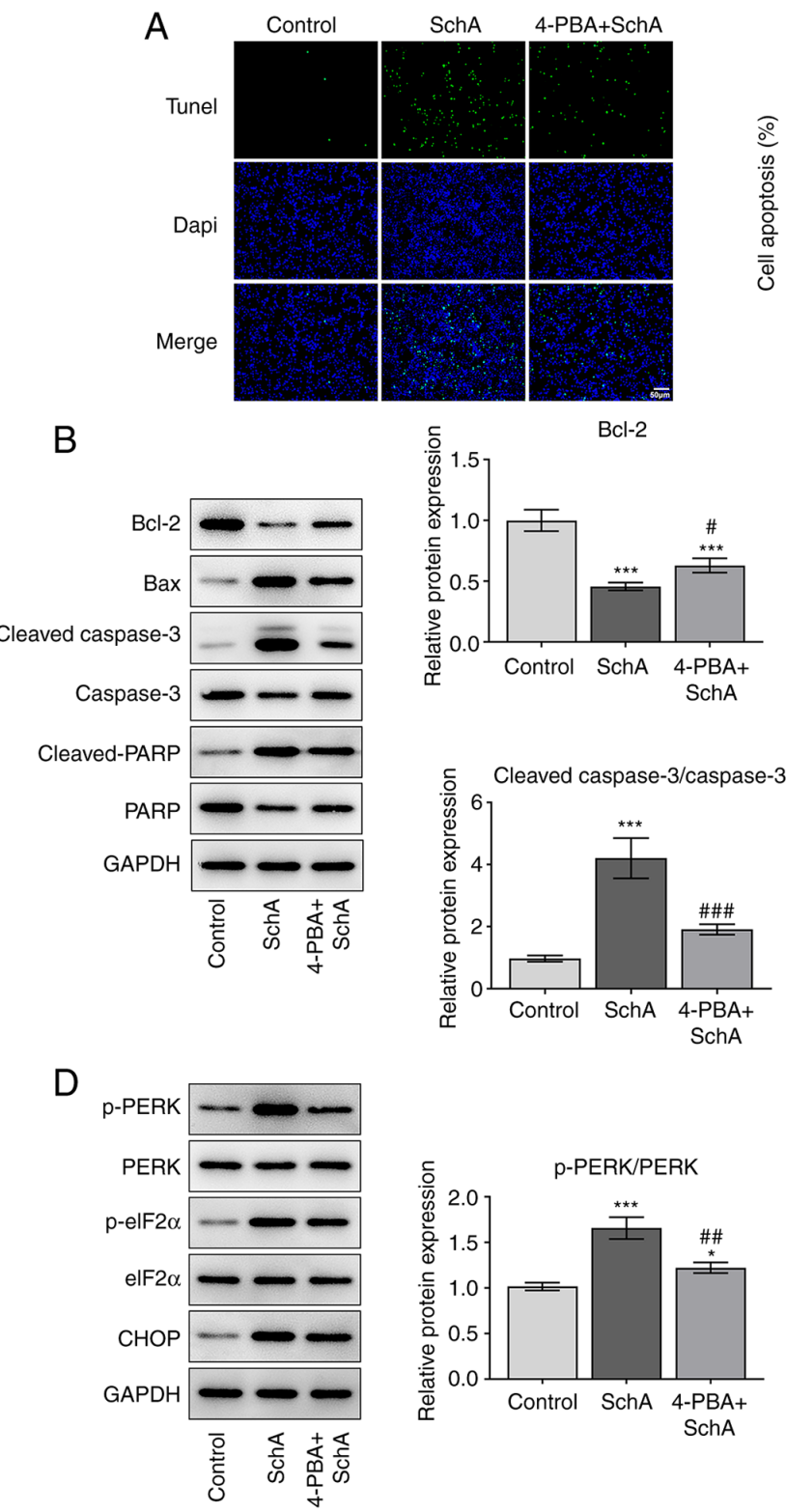
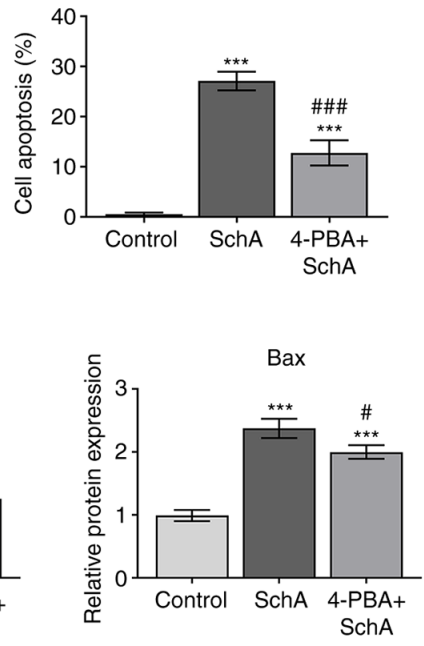

C
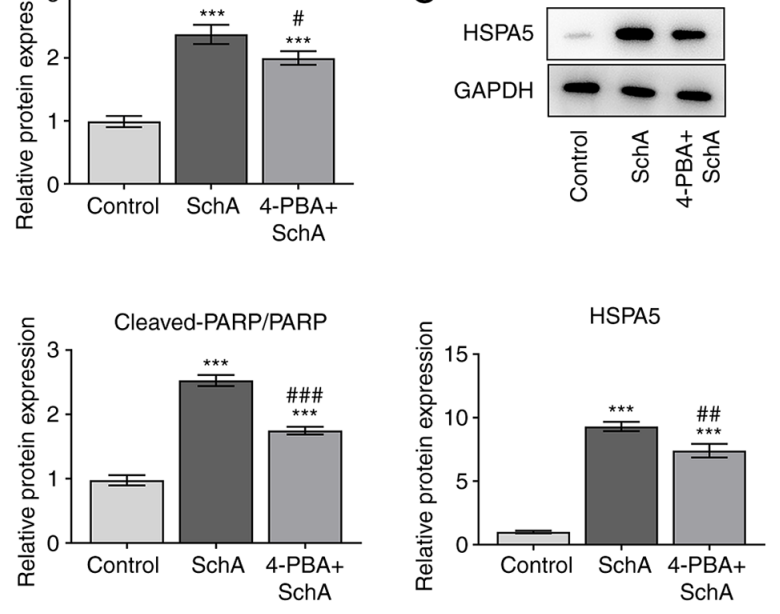

Figure 6. 4-PBA, an ER stress inhibitor, reverses the pro-apoptotic role of SchA in AGS cells. (A) The apoptosis of AGS cells after SchA and 4-PBA exposure. (B) The expression levels of apoptosis-related proteins after in AGS cells after SchA and 4-PBA exposure. (C) The expression levels of the marker of ER stress (HSPA5). (D) The expression levels of markers related to ER stress. ${ }^{*} \mathrm{P}<0.05$ and ${ }^{* * * *} \mathrm{P}<0.001$ vs. Control; ${ }^{\#} \mathrm{P}<0.05$, ${ }^{\# \#} \mathrm{P}<0.01,{ }^{\# \# \#} \mathrm{P}<0.001$ vs. SchA SchA, schizandrin A; 4-PBA, 4-phenylbutyric acid; ER, endoplasmic reticulum; HSPA5, heat shock protein family A (Hsp70) member 5; p-, phosphorylated; eiF2 $\alpha$, eukaryotic initiation factor $2 \alpha$; PARP, poly(ADP-ribose) polymerase.

\section{Funding}

This study was supported by the Interventional study of Yang Yin Jian Pi San Du Method for advanced gastric cancer (grant no. YB2017056).

\section{Availability of data and materials}

The datasets used and/or analyzed during the current study are available from the corresponding author on reasonable request.

\section{Authors' contributions}

HP designed the present study and wrote the manuscript. HP, QQ, FW and MG performed the experiments and analyzed the data. XG conceived and supervised the study and co-wrote the manuscript. XG and HP confirm the authenticity of all the raw data. All authors have read and approved the final manuscript.

\section{Ethics approval and consent to participate}

Not applicable.

\section{Patient consent for publication}

Not applicable.

\section{Competing interests}

The authors declare they have no competing interests. 


\section{References}

1. Shomali N, Mansoori B, Mohammadi A, Shirafkan N, Ghasabi M and Baradaran B: MiR-146a functions as a small silent player in gastric cancer. Biomed Pharmacother 96: 238-245, 2017.

2. Howson CP, Hiyama T and Wynder EL: The decline in gastric cancer: Epidemiology of an unplanned triumph. Epidemiol Rev 8: 1-27, 1986.

3. Van Cutsem E, Moiseyenko VM, Tjulandin S, Majlis A, Constenla M, Boni C, Rodrigues A, Fodor M, Chao Y, Voznyi E, et al: Phase III study of docetaxel and cisplatin plus fluorouracil compared with cisplatin and fluorouracil as first-line therapy for advanced gastric cancer: A report of the V325 study group. J Clin Oncol 24: 4991-4997, 2006.

4. Xu X, Rajamanicham V, Xu S, Liu Z, Yan T, Liang G, Guo G, Zhou $\mathrm{H}$ and Wang Y: Schisandrin A inhibits triple negative breast cancer cells by regulating Wnt/ER stress signaling pathway. Biomed Pharmacother 115: 108922, 2019.

5. Yan H and Guo M: Schizandrin A inhibits cellular phenotypes of breast cancer cells by repressing miR-155. IUBMB Life 72: $1640-1648,2020$

6. Chen BC, Tu SL, Zheng BA, Dong QJ, Wan ZA and Dai QQ: Schizandrin A exhibits potent anticancer activity in colorectal cancer cells by inhibiting heat shock factor 1 . Biosci Rep 40 BSR20200203, 2020.

7. Bi Y, Fu Y, Wang S, Chen X and Cai X: Schizandrin A exerts anti-tumor effects on A375 cells by down-regulating H19. Braz J Med Biol Res 52: e8385, 2019.

8. Ding Q, Li X, Sun Y and Zhang X: Schizandrin A inhibits proliferation, migration and invasion of thyroid cancer cell line TPC-1 by down regulation of microRNA-429. Cancer Biomark 24 497-508, 2019

9. Ma YY, Di ZM, Cao Q, Xu WS, Bi SX, Yu JS, Shen YJ, Yu YQ, Shen YX and Feng LJ: Xanthatin induces glioma cell apoptosis and inhibits tumor growth via activating endoplasmic reticulum stress-dependent CHOP pathway. Acta Pharmacol Sin 41: 404-414, 2020

10. Xian H, Feng W and Zhang J: Schizandrin A enhances the efficacy of gefitinib by suppressing IKK $\beta / N F-\kappa B$ signaling in non-small cell lung cancer. Eur J Pharmacol 855: 10-19, 2019.

11. Urra H, Dufey E, Avril T, Chevet E and Hetz C: Endoplasmic reticulum stress and the hallmarks of cancer. Trends Cancer 2 : 252-262, 2016.

12. Farina $P$, Tabouret E, Lehmann $P$, Barrie M, Petrirena G, Campello C, Boucard C, Graillon T, Girard N and Chinot O: Relationship between magnetic resonance imaging characteristics and plasmatic levels of MMP2 and MMP9 in patients with recurrent high-grade gliomas treated by Bevacizumab and Irinotecan. J Neurooncol 132: 433-437, 2017.

13. Shi Y, Shi H, Zhang B, Yan Y, Han X, Jiang W, Qian H and Xu W: miR-373 suppresses gastric cancer metastasis by downregulating vimentin. Mol Med Rep 17: 4027-4034, 2018.

14. Wu D, Zhang P, Ma J, Xu J, Yang L, Xu W, Que H, Chen M and $\mathrm{Xu} \mathrm{H}$ : Serum biomarker panels for the diagnosis of gastric cancer. Cancer Med 8: 1576-1583, 2019.

15. Rona KA, Schwameis K, Zehetner J, Samakar K, Green K, Samaan J, Sandhu K, Bildzukewicz N, Katkhouda N and Lipham JC: Gastric cancer in the young: An advanced disease with poor prognostic features. J Surg Oncol 115: 371-375, 2017.

16. Liu X, Xiu LJ, Jiao JP, Zhao J, Zhao Y, Lu Y, Shi J, Li YJ, Ye M, Gu YF, et al: Traditional Chinese medicine integrated with chemotherapy for stage IV non-surgical gastric cancer: A retrospective clinical analysis. J Integr Med 15: 469-475, 2017.

17. Lee K, Ahn JH,Lee KT, Jang DS and Choi JH: Deoxyschizandrin, isolated from schisandra berries, induces cell cycle arrest in ovarian cancer cells and inhibits the protumoural activation of tumour-associated macrophages. Nutrients 10: 91, 2018.
18. Panossian A and Wikman G: Pharmacology of Schisandra chinensis Bail.: An overview of Russian research and uses in medicine. J Ethnopharmacol 118: 183-212, 2008.

19. Li L, Pan Q, Sun M, Lu Q and Hu X: Dibenzocyclooctadiene lignans: A class of novel inhibitors of multidrug resistanceassociated protein 1. Life Sci 80: 741-748, 2007.

20. Chen $X$ and Cubillos-Ruiz JR: Endoplasmic reticulum stress signals in the tumour and its microenvironment. Nat Rev Cancer 21: 71-88, 2021.

21. Bettigole SE and Glimcher LH: Endoplasmic reticulum stress in immunity. Annu Rev Immunol 33: 107-138, 2015.

22. Wang S and Kaufman RJ: The impact of the unfolded protein response on human disease. J Cell Biol 197: 857-867, 2012.

23. Bertolotti A, Zhang Y, Hendershot LM, Harding HP and Ron D: Dynamic interaction of BiP and ER stress transducers in the unfolded-protein response. Nat Cell Biol 2: 326-332, 2000.

24. Mohamed E, Cao Y and Rodriguez PC: Endoplasmic reticulum stress regulates tumor growth and anti-tumor immunity: A promising opportunity for cancer immunotherapy. Cancer Immunol Immunother 66: 1069-1078, 2017.

25. Wang J, Hu X and Jiang H: ERS-PERK signaling pathway-mediated Nrf2/ARE-HO-1 axis: A novel therapeutic target for attenuating myocardial ischemia and reperfusion injury. Int $\mathrm{J}$ Cardiol 203: 779-780, 2016.

26. Sozen E, Karademir B and Ozer NK: Basic mechanisms in endoplasmic reticulum stress and relation to cardiovascular diseases. Free Radic Biol Med 78: 30-41, 2015.

27. Oakes SA and Papa FR: The role of endoplasmic reticulum stress in human pathology. Annu Rev Pathol 10: 173-194, 2015.

28. Kaira K, Toyoda M, Shimizu A, Shino M, Sakakura K, Takayasu Y, Takahashi K, Asao T and Chikamatsu K: Expression of ER stress markers (GRP78/BiP and PERK) in adenoid cystic carcinoma. Acta Otolaryngol 136: 1-7, 2016.

29. Zheng YZ, Cao ZG, Hu X and Shao ZM: The endoplasmic reticulum stress markers GRP78 and CHOP predict disease-free survival and responsiveness to chemotherapy in breast cancer. Breast Cancer Res Treat 145: 349-358, 2014.

30. Nagelkerke A, Bussink J, Sweep FC and Span PN: The unfolded protein response as a target for cancer therapy. Biochim Biophys Acta 1846: 277-284, 2014

31. Moon SY, Kim HS, Nho KW, Jang YJ and Lee SK: Endoplasmic reticulum stress induces epithelial-mesenchymal transition through autophagy via activation of c-Src kinase. Nephron Exp Nephrol 126: 127-140, 2014.

32. Wang $M$ and Kaufman RJ: The impact of the endoplasmic reticulum protein-folding environment on cancer development. Nat Rev Cancer 14: 581-597, 2014.

33. Li Y, Liu H, Huang YY, Pu LJ, Zhang XD, Jiang CC and Jiang ZW: Suppression of endoplasmic reticulum stress-induced invasion and migration of breast cancer cells through the downregulation of heparanase. Int J Mol Med 31: 1234-1242, 2013

34. Liu R, Li X, Gao W, Zhou Y, Wey S, Mitra SK, Krasnoperov V, Dong D, Liu S, Li D, et al: Monoclonal antibody against cell surface GRP78 as a novel agent in suppressing PI3K/AKT signaling, tumor growth, and metastasis. Clin Cancer Res 19: 6802-6811, 2013 .

35. Dong KN, Huang X, Xing WY, Guo WW and Feng R: Effect of endoplasmic reticulum stress on gastric cancer cell migration and invasion. World Chin J Dig 24: 1485, 2016.

This work is licensed under a Creative Commons Attribution-NonCommercial-NoDerivatives 4.0 International (CC BY-NC-ND 4.0) License. 